\title{
Langage de la critique, critique du langage
}

La transition postmoderne

Criticism Language, Criticism of Language. The Postmodern Transition

Jacques Lucan

\section{(2) OpenEdition}

Journals

Édition électronique

URL : http://journals.openedition.org/crau/309

DOI : $10.4000 /$ crau.309

ISSN : 2547-5746

Éditeur

Éditions du patrimoine

Édition imprimée

Date de publication : 1 décembre 2009

Pagination : 113-120

ISBN : 978-2-85822-944-4

ISSN : 1296-4077

Référence électronique

Jacques Lucan, "Langage de la critique, critique du langage », Les Cahiers de la recherche architecturale et urbaine [En ligne], 24/25 | 2009, mis en ligne le 01 septembre 2017, consulté le 03 mai 2019. URL:

http://journals.openedition.org/crau/309; DOI : 10.4000/crau.309 
L'article de Jacques Lucan pose ici une hypothèse: après le Mouvement moderne, avec le reflux qu'a pu représenter le postmodernisme, la crise est celle du langage architectural. En effet, dans les années 1970, au-delà de leurs différences ou même de leurs oppositions, ce qui rassemble des protagonistes comme Robert Venturi, Manfredo Tafuri, Aldo Rossi et Peter Eisenman, par exemple, est une commune préoccupation concernant les fondements de l'architecture comme langage. Cette préoccupation mène à une interrogation sur le régime de l'œuvre d'art contemporaine, c'est-à-dire, en particulier pour ce qui nous intéresse, sur les règles qu'établit pour lui-même le projet architectural, règles intrinsèques que la critique architecturale doit tenter d'expliciter.

\section{Langage de la critique, critique du langage}

La transition postmoderne

JACQUES LUCAN

En 1969, Michel Foucault posait la question : "Comment se fait-il qu'à une époque donnée on puisse dire ceci et que jamais cela n'ait été dit $^{1}$ ? " C'était au moment de la publication de L'Archéologie du savoir, livre dans lequel était posée une question presque identique: "Comment se fait-il que tel énoncé soit apparu et nul autre à sa place $^{2}$ ? »Ces questions valaient pour divers domaines de connaissance ou de compétence; par inférence, posons qu'elles valent aussi pour l'architecture. Mais si ces questions s'adressaient bien sûr à I'histoire, on pouvait aisément penser qu'elles s'adressaient aussi au présent. Posons donc que l'activité historienne est rejointe par I'activité critique.

À la fin des années 1960 et au début des années 1970, que l'on se tournât vers I'Italie avec Aldo Rossi et Manfredo Tafuri, par exemple, ou vers les États-Unis avec Robert Venturi et Peter Eisenman, autres exemples particulièrement contrastés, l'enjeu était une reconquête disciplinaire, qui ressemblera quelquefois à un repli disciplinaire. La reconquête disciplinaire était corrélative d'une activité critique - ce à quoi s'employaient les quatre protagonistes ici nommés. Elle signifiait que l'architecture était envisagée à l'égal d'un énoncé dont on

1. Michel Foucault, «La naissance d'un monde », entretien avec JeanMichel Palmier, Le Monde, supplément: Le Monde des livres, $n^{\circ}$ 7558, 3 mai 1969, repris dans Dits et écrits 1954-1988, vol. I, 1954-1969, Paris, Gallimard, 1994, p. 787.

2. M. Foucault, L'Archéologie du savoir, Paris, Gallimard, 1969, p. 39. 


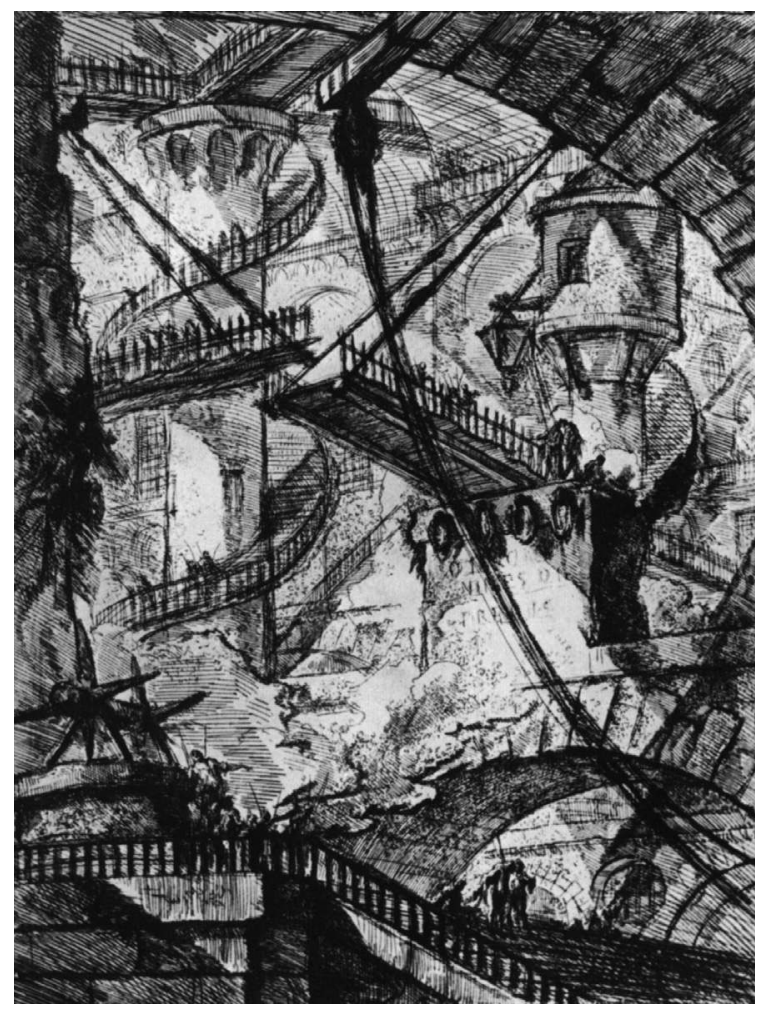

Giovan Battista Piranesi, planche VII (" premier état ") de Carceri d'Invenzione, 1761. Figure 9 de Manfredo Tafuri, La Sfera e il Labirinto. Avanguardie et architettura da Piranesi agli anni'70, 1980. devait comprendre la logique, la structure - nous étions aux lendemains de l'apogée du structuralisme -, ce que plus tard, à la suite de Jacques Derrida, certains appelleront déconstruction, dont le philosophe disait en 1967 : "Les mouvements de déconstruction ne sollicitent pas les structures du dehors. Ils ne sont possibles et efficaces, ils n'ajustent leurs coups qu'en habitant ces structures. En les habitant d'une certaine manière, car on habite toujours et plus encore quand on ne s'en doute pas ${ }^{3}$. »

À la reconquête ou au repli disciplinaire correspondait une inquiétude relative au langage, la métaphore langagière s'appliquant souvent à l'architecture sans autre procès. Posons maintenant que l'inquiétude relative au langage a renouvelé les approches possibles de la critique architecturale. Remarquons aussi que les propos précédents butent sur une aporie: d'un côté, il s'agit de solliciter des structures, donc de se préoccuper de permanence ou de "longue durée ", pour reprendre les mots de Fernand Braudel; d'un autre côté, il s'agit de comprendre le nouveau, que l'« on puisse dire ceci et que jamais cela n'ait été dit ». C'est à cette aporie que la critique est toujours confrontée.

\section{Une même préoccupation langagière: Venturi, Tafuri, Rossi et Eisenman}

Le travail engagé par Venturi avec son livre paru en 1966, Complexity and Contradiction in Architecture, s'appuie sur l'analyse d'éléments d'architecture, en cherchant à expliciter leurs relations, en particulier les complexités et les contradictions de celles-ci. Venturi trouve notamment un intérêt à toutes les "déviations " maniéristes, c'est-àdire à des figures syntaxiques qui s'apparentent souvent,
3. Jacques Derrida, De la grammatologie, Paris, Éditions de Minuit, 1967, p. 39.

4. Robert Venturi, De l'ambiguïté en architecture, Paris, Dunod, 1971 (trad. de Complexity and Contradiction in Architecture, New York, The Museum of Modern Art, 1966), p. 22.

5. Ibid., p. 51.

6. Manfredo Tafuri, «Per una critica dell'ideologia architettonica », Contropiano, n० 1, 1969, p. 38, repris et développé dans M. Tafuri, Progetto e utopia. Architettura e sviluppo capitalistico, Rome-Bari, Laterza, 1973 (trad. fr.: Projet et utopie, de l'avantgarde à la métropole, Paris, Dunod, 1979).

7. Ibid., p. 39

8. Le concept d'hétérotopie doit sans doute à Michel Foucault dont l'article « Des espaces autres: utopies et hétérotopies » était paru en français (sous le nom de Marcel Foucault) dans L'Architettura, cronache e storia (vol. XIII, n' 12, avril 1968); cet article est le texte d'une conférence faite à Paris devant le Cercle d'études architecturales le 14 mars 1967 (pour une nouvelle publication, voir M. Foucault, op. cit. note 1, vol. IV, 1980-1988). Pour Foucault, les « espaces autres » sont les prisons, cimetières, maisons closes, etc., et «l'hétérotopie a le pouvoir de juxtaposer en un seul lieu réel plusieurs espaces, plusieurs emplacements qui sont en eux-mêmes incompatibles ». 
pour un regard «moderne », à des collages. II se préoccupe de reconnaître une multiplicité de dispositifs dans lesquels des éléments peuvent avoir une double fonction ou une double signification, et il soumet l'architecture moderne au même traitement que les architectures qu'il emprunte à une histoire plus ancienne. Il entend donc ne plus se laisser intimider par ce qu'il juge avoir été «la morale et le langage puritains de l'architecture moderne orthodoxe ${ }^{4}$. II fait siens aussi bien le tropisme du langage poétique, qui veut que les mots soient les vecteurs d'associations ou de correspondances nouvelles et inattendues, que l'attitude des protagonistes du pop art qui « donnent un sens inhabituel à des éléments communs en changeant leur contexte ou en augmentant leurs dimensions ${ }^{5}$ ».

Même si Tafuri apprécie peu Venturi, il adopte l'analogie langagière. Dans cette optique, Piranèse est pour lui une clef de lecture paradoxale, le Champ de Mars étant chargé d'une valeur prophétique. Dans le Champ de Mars, en effet, "chaque forme dérivée du classicisme est traitée comme un pur fragment, comme symbole dénaturé, comme lambeau halluciné d'un "ordre" en décomposition [macerazione] $]^{6}$ ». II est à rapprocher d'un " bricolage colossal ${ }^{7}$ " qui fait perdre toute unité à la totalité et, à son propos, Tafuri introduit le concept foucaldien d'hétérotopie ${ }^{8}$. Quant aux Prisons, ce sont des « compositions "impossibles" ". Si les Prisons illustrent donc de multiples façons la « crise de l'objet architectural ${ }^{10}$ ", si elles sont, en acte, une « critique systématique du concept de lieu ${ }^{11}$ ", « du concept de "centre"12 ", « du concept d'espace ${ }^{13}$ ", le Champ de Mars est quant à lui le " triomphe du fragment ${ }^{14}$ ". Prisons et Champ de Mars complètent leurs «messages » : « Les Prisons et le Champ de Mars mettent définitivement en crise le "langage comme principe d'action sur le monde" ${ }^{15}$. »

Tafuri lit dans les œuvres de Piranèse le drame d'un temps qui a déjà quitté l'horizon de l'harmonie classique et de l'organicité de la forme, qui ne peut plus en appeler à un idéal de totalité, qui en a fini avec les concepts d'Alberti, concinnitas et finitio, et exalte un divorce entre les signes architecturaux et leurs signifiés. Pour Tafuri, I'hétérotopie piranésienne est ainsi à l'image du temps présent: le Champ de Mars est un ensemble de fragments hétérogènes ayant perdu toute possibilité de signification collective, du moment que ces fragments n'appartiennent plus à une ville entendue comme « lieu de la Forme ${ }^{16}$ ".

Aldo Rossi, selon Tafuri, à travers des projets pour la plupart seulement dessinés, "en présentant une syntaxe de signes vides, d'exclusions programmées, de rigoureuses limitations, révèle la nature inflexible de l'arbitraire et la fausse dialectique entre liberté et norme qui sont les caractéristiques de l'ordre linguistique ${ }^{17}$. „Plus tard, Tafuri et Francesco Dal Co ajouteront que Rossi situe son travail, à l'instar de Louis I. Kahn, dans " la nostalgie d'un ordre ancestral du langage ${ }^{18}$ », dans la réappropriation d'un alphabet, dans " une recherche méthodique du lieu où la forme a pu retrouver l'usage de la langue 19 ".

En 1979, lors de la seconde exposition d'œuvres de Rossi organisée par l'Institute for Architecture and Urban Studies (IAUS) à New York, Peter Eisenman analyse le travail de l'architecte en commençant par commenter un dessin dédié à Tafuri, L'Architecture assassinée (1975).
9. M. Tafuri, Progetto e utopia, op. cit. note 6, p. 21.

10. M. Tafuri, " "L'architetto scellerato": G. B. Piranesi, l'eterotopia et il viaggio ", dans La Sfera e il Labirinto. Avanguardie e architettura da Piranesi agli anni'70, Turin, Giulio Einaudi Editore, 1980, p. 42 (première version partielle en traduction française: " Giovan Battista Piranesi : I'utopie négative dans I'architecture ", L'Architecture d'aujourd'hui, n 184, mars-avril 1976).
11. Ibid., p. 36.

12. Ibid., p. 37

13. Ibid., p. 38

14. Ibid., p. 48.

15. Ibid., p. 47.

16. Ibid., p. 49
17. M. Tafuri, «L'Architecture dans le Boudoir: The Language of Criticism and the Criticism of Language ", Oppositions, n³, mai 1974 (repris et complété dans La Sfera e il Labirinto, op. cit. note 10), p. 42.

18. Francesco Dal Co et M. Tafuri, Architecture contemporaine, Paris/Milan, Gallimard/Electa, 1991 (traduction de Architettura contemporanea, Milan, Electa, 1976), p. 379. 
Ce dessin met en scène une dislocation de bâtiments - certains fragments évoquant des projets de l'architecte milanais -, comme s'ils étaient victimes d'un tremblement de terre. Pour Eisenman, L'Architecture assassinée est " analogue aux "déconstructions" de Piranèse ${ }^{20}$ ". La boucle est bouclée.

Les préoccupations d'Eisenman visent la compréhension de ce qu'il nomme lui-même des "systèmes formels ». En venant en Grande-Bretagne, il avait rencontré Colin Rowe, auquel il avait emprunté l'idée d'une transparence virtuelle comme concept de compréhension de certains dispositifs de l'architecture moderne. Avec Rowe, il était allé à Côme en 1961 voir l'œuvre de Giuseppe Terragni, dont il analysera la Casa del Fascio $(1932-1936)^{21}$ et la Casa Giuliani Frigerio (1939-1940) 22 selon des principes de stratification spatiale. À cette occasion, il avait construit « une hypothèse sommaire à propos des aspects syntaxiques de la forme architecturale $^{23}$ ", s'inspirant des travaux du linguiste Noam Chomsky. Son doctorat en philosophie obtenu à Cambridge, en 1963, analyse des œuvres de Le Corbusier et Pierre Jeanneret, de Frank Lloyd Wright, d'Alvar Aalto et de Terragni. Il cherche à distinguer des systèmes, sans que les paramètres constructifs et fonctionnels ${ }^{24}$ soient prévalents. Pour Eisenman, un système formel est en deçà des circonstances particulières d'un projet, et c'est lui qui détermine consciemment ou inconsciemment la forme spécifique d'un bâtiment. L'architecture doit donc être appréhendée d'un point de vue syntaxique ou conceptuel, les deux mots étant souvent quasiment synonymes. L'insistance syntaxique met de côté toute dimension sémantique, les œuvres devant être regar-

\section{Ibid.}

20. Peter Eisenman, «The House of the Dead as the City of Survival ", Aldo Rossi in America: 1976 to 1979, New York, The Institute for Architecture and Urban Studies, IAUS Exhibition Catalogue, catalogue 2 s.d. 1979, p. 4.

21. Voir P. Eisenman, « Dall'oggetto alla relazionalità: la casa del Fascio di Terragni/ From Object to Relationship: The Casa

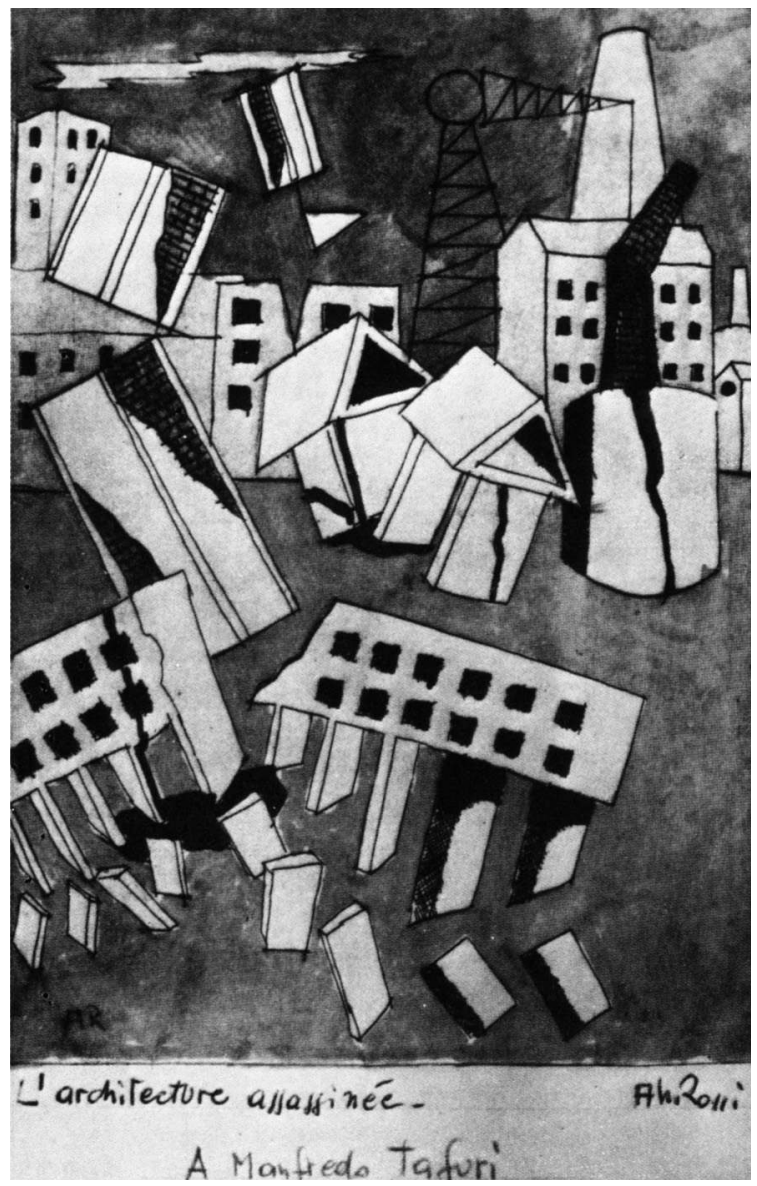

Aldo Rossi, L'Architecture assassinée. À Manfredo Tafuri, dessin publié dans Aldo Rossi in America: 1976 to 1979, The Institute for Architecture and Urban Studies. IAUS Exhibition Catalogue, catalogue 2 (1979), p. 4.

23. Ibid., p. 38. Les études sur Terragni sont reprises dans P. Eisenman, Giuseppe Terragni: Transformations, Décompositions, Critiques, New York, The Monacelli Press, 2003, avec un texte de Manfredo Tafuri, "Subject and Mask » (une première fois publié dans Oppositions, n 11, hiver 1977).

24. Voir P. Eisenman, The Formal Basis of Modern Architecture [Cambridge, 1963], Baden, Lars Müller Publishers, 2006 (publication tardive de la thèse soutenue 
dées en elles-mêmes et pour elles-mêmes. C'est à ce moment qu'Eisenman salue la peinture « hard-edge » et le travail des artistes minimalistes, notamment Donald Judd et Robert Morris, et qu'il fait explicitement référence à la fameuse phrase de Barbara Rose, qui décrit les œuvres de ces derniers: «La chose n'est ainsi pas censée "signifier", semble-t-il, autre chose que ce qu'elle est: c'est-à-dire qu'elle n'est pas censée suggérer autre chose qu'elle-même ${ }^{25}$. »Ce repli sur la chose même confirme l'inscription des préoccupations d'Eisenman dans un horizon linguistique autonome, le propos de Rose faisant indubitablement écho à la dernière et célèbre phrase attribuée à Ferdinand de Saussure, conclusive de son Cours de linguistique générale: " La linguistique a pour unique et véritable objet la langue envisagée en ellemême et pour elle-même ${ }^{26}$. »
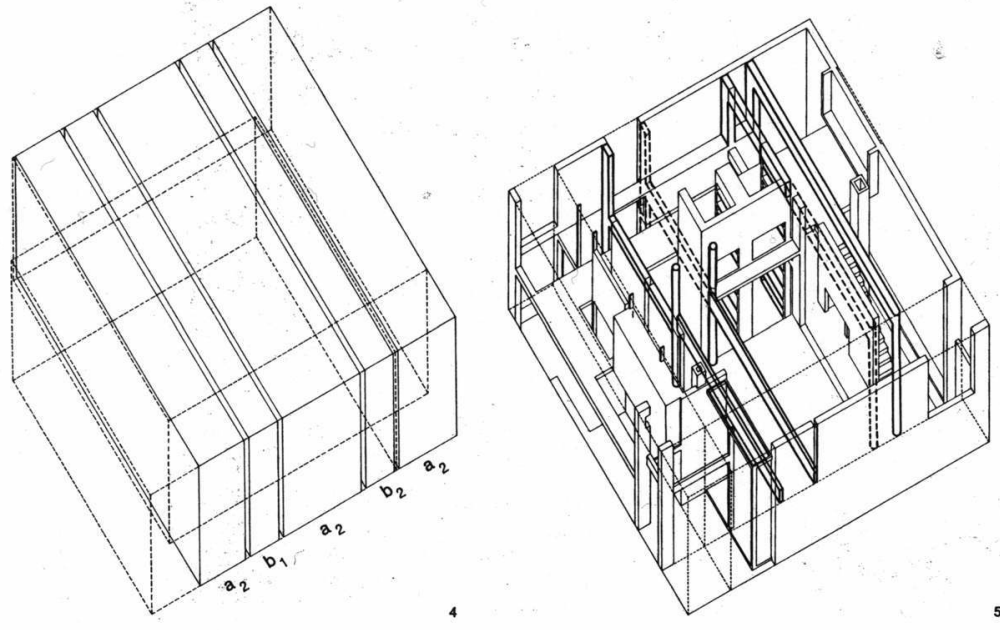

Peter Eisenman, « House I, 1967 », suite d'opérations formelles pour la conception de la maison. Figure extraite de Five Architects: Eisenman, Graves, Gwathmey, Hejduk, Meier [1972], New York, 1975, p. 22.

en 1963 au Trinity College de l'université de Cambridge; des éléments en sont repris dans "Towards an Understanding of Form in Architecture ", Architectural Design, $n^{\circ} 10$, octobre 1963), p. 25 et 26.

25. Barbara Rose, "ABC Art », Art in America, octobre-novembre 1965, repris dans Gregory Battcock (dir.), Minimal Art. A Critical Anthology [1968], Berkeley et Los Angeles, University of California Press, 1995 (traduction française dans Claude Gintz [dir.],
Regards sur l'art américain des années soixante, Paris, Territoires, 1979), p. 291. Eisenman dit des œuvres de Judd et Morris, dans « Notes on conceptual architecture. Towards a Definition », Design Quaterly, n 78-79, 1970 : « Les objets n'ont pas de signification autre que celle des objets en eux-mêmes » (repris dans P. Eisenman, Eisenman Inside Out: Selected Writings, 1963-1988, New Haven et Londres, Yale University Press, 2004, p. 13).

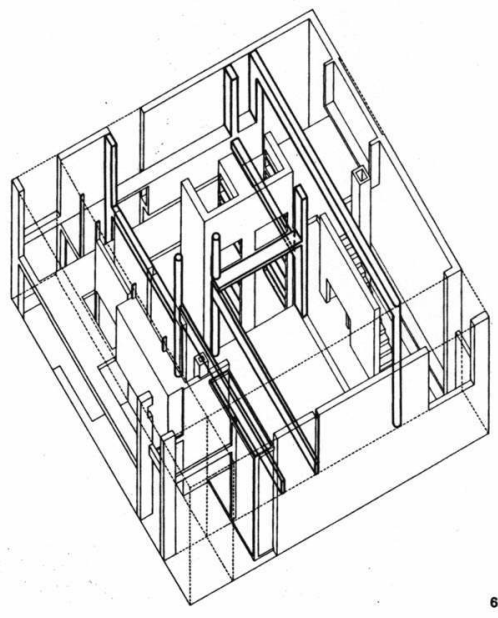

26. Ferdinand de Saussure, Cours de linguistique générale [1916], Paris, Payot, 1972 , p. 317 (sur l'attribution sans doute fallacieuse de cette phrase à Saussure, voir: ibid., note 305, p. 476-477). 


\section{Transition postmoderne}

Bien qu'ils aient des préoccupations différentes et qu'ils puissent nous sembler tirer l'architecture dans des voies absolument divergentes, Venturi, Tafuri, Rossi et Eisenman ont en commun une même préoccupation langagière. Pour rendre intelligible cette commune préoccupation, j'ai en quelque sorte construit une intrigue - en suivant ici la définition qu'en donne Paul Ricœur: "L'intrigue est l'unité narrative de base qui compose [des] ingrédients hétérogènes dans une totalité intelligible ${ }^{27}$.»

La construction de cette intrigue est du ressort de I'histoire et de la critique architecturales. Elle nous permet d'appréhender rétrospectivement les années 1970 comme traversées par une ligne de force, alors qu'à un premier regard il pourrait sembler qu'elles ne faisaient que tirer à hue et à dia. La construction de cette intrigue est une hypothèse de compréhension d'une période de crise qui a vu se développer les interrogations sur la signification même des éléments et des dispositifs architecturaux, sur leur possible ou impossible cohérence, en deçà ou au-delà des exigences relatives aux conditions fonctionnelles ou économiques, qui furent des critères dominants pour les années " modernes » d'après la Seconde Guerre mondiale.

II n'est pas surprenant que, dans cette période de crise, l'inquiétude soit relative au langage. Cette inquiétude sera canalisée selon plusieurs voies. La plus dominante verra l'emprise de l'historicisme s'étendre de façon extraordinaire, qu'il soit architectural ou urbain. L'historicisme architectural - dont le chantre est
Charles Jencks - produit une diversité éclectique qui n'a rien à envier à celle de la seconde moitié du $X I X e$ siècle, sinon une qualité constructive le plus souvent perdue. L'historicisme urbain - dont les représentants les plus radicaux sont les frères Léon et Robert Krier - cherche à renouer avec des formes qui constitueraient un cadre pour que les bâtiments individuels aient une signification collective; I'historicisme urbain, dans bien des cas, produit des images nostalgiques, sinon kitsch ou populistes, images auxquelles sont particulièrement sensibles les commanditaires publics et privés, en France et dans bien d'autres pays, pas seulement européens.

La période de crise est celle d'un passage, le passage à la condition postmoderne, comme I'appelait JeanFrançois Lyotard ${ }^{28}$, passage qui a pu être négocié - sans " baisser les bras » - grâce à Rem Koolhaas et Jean Nouvel, Herzog et de Meuron, Toyo Ito et Kazuyo Sejima, parmi d'autres. Ce passage a bouleversé le paysage même de la production architecturale, la condition postmoderne étant corrélative d'une mondialisation de la production architecturale, une mutation qui suscite des questions auxquelles est confrontée la critique architecturale - et que l'on ne peut faire ici que poser. Par exemple, l'architecture mondialisée est-elle un nouvel avatar d'un style international? Est-il encore possible de construire aujourd'hui un rempart analogue à celui qu'avait voulu être l'hypothèse du « régionalisme critique " énoncée par Kenneth Frampton au début des années 1980? Est-il encore possible de parler d'architecture " de résistance » comme d'aucuns l'ont fait au même moment?
27. Paul Ricœur, Du texte à l'action. Essais d'herméneutique, II, Paris, Le Seuil, 1986, p. 15.
28. Voir Jean-François Lyotard, La Condition postmoderne, Paris, Éditions de Minuit, 1979. 


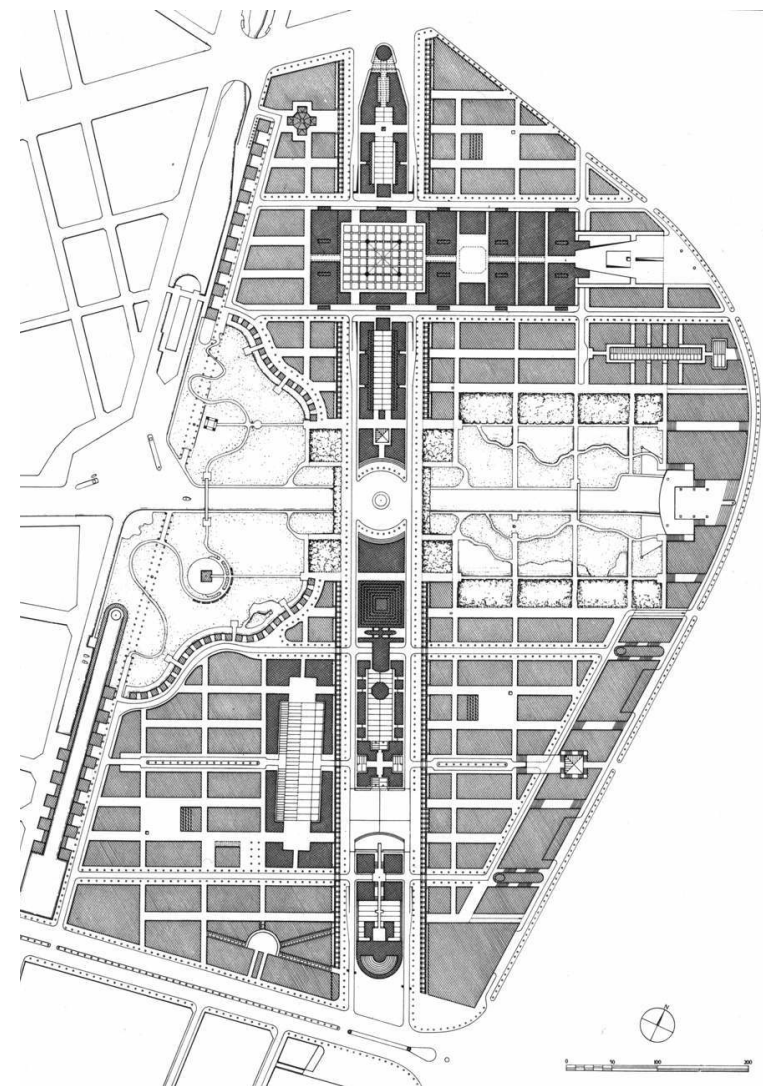

Léon Krier, projet de concours pour l'aménagement des terrains des anciens abattoirs de La Villette (1976), Paris. Plan d'ensemble.

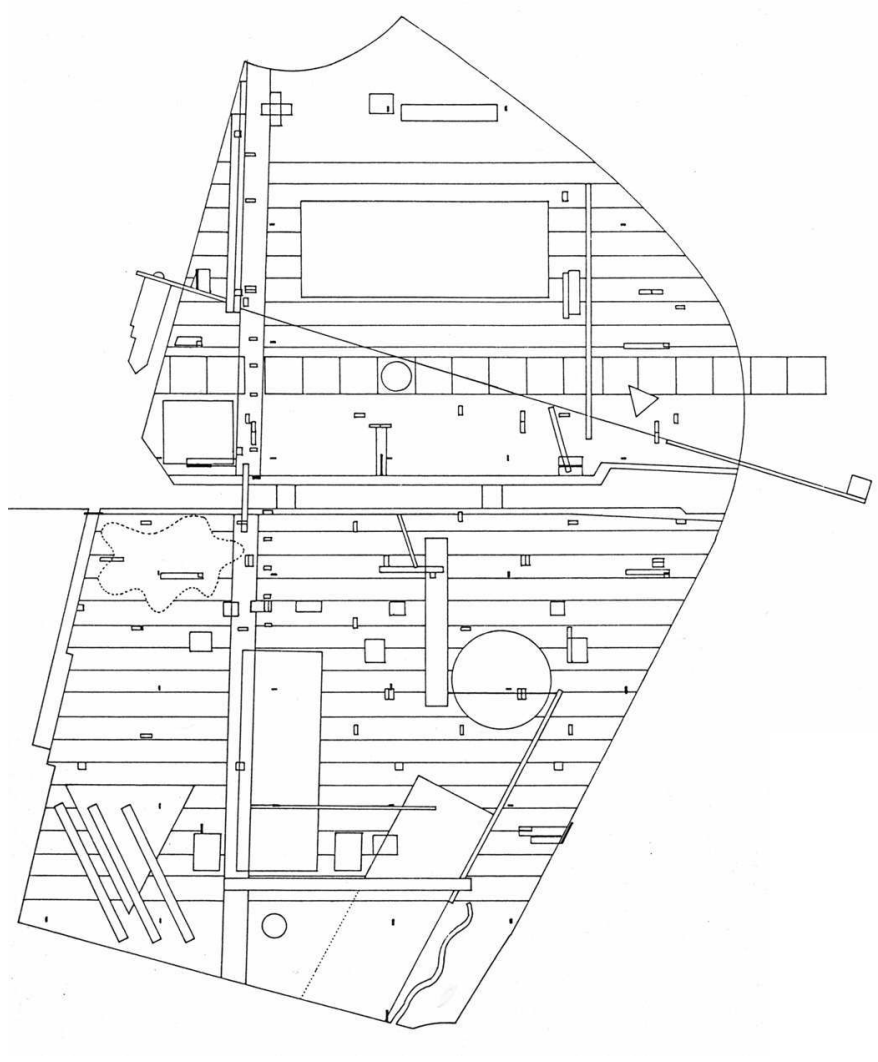

OMA-Rem Koolhaas, projet de concours pour le Parc de La Villette (1982-1983), Paris. Plan d'ensemble. 


\section{Règles}

L'inquiétude relative au langage s'est-elle éteinte? Avançons I'hypothèse qu'un travail architectural doit être à même de définir ce qu'il en est de ses propres règles. Après la Seconde Guerre mondiale, Le Corbusier appelait cela « le jeu », jeu qui constituait la spécificité ultime du travail architectural, et qui était en deçà ou au-delà de la réponse adéquate à un programme fonctionnel. II précisait ainsi dans une note manuscrite inédite du

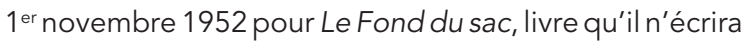
pas: "Quand mon client me remplit la tête de ses petits besoins j'accepte jusqu'à un certain point où je dis non, impossible! Car c'est alors hors de la règle de mon jeu, du jeu en question, le jeu de cette maison, de cette combinaison dont la règle a surgi avec l'heure de création, s'est développée, affirmée, devenue maîtresse. Tout à l'intérieur de la règle! Rien hors la règle. Sinon je n'ai plus de raison d'exister. »

La raison d'exister réside donc dans le jeu et sa règle. Mais cette règle n'est pas extérieure et le projet ne fait pas que l'appliquer. Non, la règle s'institue dans le moment même de la création, « avec I'heure de création », comme le dit Le Corbusier. Lyotard dit du texte de l'écrivain ou de l'œuvre de l'artiste postmodernes qu'ils ne sont pas gouvernés par des règles déjà établies, mais bien au contraire que celles-ci sont « ce que l'œuvre ou le texte recherche ", I'artiste ou l'écrivain travaillant donc "sans règles, et pour établir les règles de ce qui aura été fait $^{29}$ ». Thierry de Duve, quant à lui, dit que « le travail formel d'un artiste [...] ne saurait obéir à des règles préexistantes mais il n'est pas sans règles. Il les institue dans le mouvement même par lequel il les interroge ${ }^{30}$. "
En dernière instance, tenter d'expliciter ce qu'il en est du travail d'un architecte, c'est tenter de comprendre ce qu'il en est des règles de formation de ses projets, de leur processus de conception. N'est-ce pas l'enjeu même de la critique architecturale? Et c'est bien pour cela que I'inquiétude relative au langage reste vive.

Pour prendre un exemple: en 1978, au sujet de certaines parties du projet de I'OMA (Office for Metropolitan Architecture) pour l'extension du Parlement néerlandais à La Haye, Koolhaas avait parlé d' « architecture processuelle [architecture-through-process] », c'est-àdire d'une architecture qui est générée par le développement de ce que l'on peut nommer des opérations formelles spécifiques, opérations définies à partir de considérations tant programmatiques que contextuelles, mais aussi à partir de considérations relatives à la stabilité ou l'instabilité des usages, relatives aux sensations que l'on entend faire éprouver, etc.

La conception processuelle entre bien sûr en résonance avec les préoccupations de beaucoup d'artistes contemporains. Elle entre aussi en phase avec le développement de moyens informatiques qui ne font pas que révolutionner les moyens de représentation, mais qui apportent de nouvelles possibilités de génération formelle, tout aussi bien que de nouvelles possibilités de liaisons entre conception et fabrication, conception et construction.

Dans la transition postmoderne, les divers paramètres définissant les projets architecturaux se nouent selon des modalités nouvelles, ce qui, pour la critique architecturale, rend toujours actuelle l'interrogation initiale de Foucault.

29. J.-Fr. Lyotard, Les Immatériaux, Paris,

Centre Georges-Pompidou, 1985.

30. Thierry de Duve, Nominalisme pictural,

Paris, Éditions de Minuit, 1984, p. 16. 